\title{
Recover Neuropathology
}

Can. J. Neurol. Sci. 2004; 31: 140-141

Dr. Roland Auer's editorial "Whither Neuropathology" (Can J Neurol Sci 2003; 30(4): 299-301) offers a misinformed, albeit passionate defense of Neuropathology remaining a recognized primary specialty of the Royal College of Physicians and Surgeons of Canada (RCPSC). It is a misconception that subspecialty status would result in Neuropathology losing its examination at the completion of residency training. Nor is the cost of the examination the sole motivating factor in reviewing Neuropathology as a primary specialty. Rather, Neuropathology is part of a review to ensure that: existing RCPSC primary specialties meet the approved criteria for recognition as a specialty; training models are effective; and the principle of generalism preceding specialization and subspecialization is upheld.

In 1999, the Council of the RCPSC approved a process for the review of primary specialties recommended by the Committee of Specialties, prompted by the College's removal of the moratorium on the creation of new subspecialties. A specialty is defined as having a broad-based body of knowledge that is relevant in both community and tertiary settings and is a foundation for additional competencies (subspecialties). Specialties must be able to produce a valid and reliable examination that leads to certification by the RCPSC. Training leading to specialty certification begins after successful completion of a medical degree.

A subspecialty is defined as having a body of knowledge and identifiable competencies that build upon the broad-based body of knowledge defined in a specialty. There must be evidence of societal need for the subspecialty to justify development of and support for a RCPSC accredited training program. Successful completion of a subspecialty requires certification by examination in one of the primary specialties and successful completion of a subspecialty-training program. Subspecialty training must be at least one year in duration, can only be entered in the final year of primary specialty training, and may be evaluated with or without a RCPSC examination.

The initial identification of existing specialties to be reviewed by the College includes laboratory-based disciplines (Hematological Pathology, Medical Biochemistry, Neuropathology, Medical Microbiology) as well as Medical Genetics, Occupational Medicine, Cardiac and Thoracic Surgery and was based on the number of residents within smaller RCPSC programs. This criterion was used because it is indicative of the number of residents selecting into the discipline at their PGY-1 year (entry from medical school) and because of the difficulties associated with providing a valid and reliable examination for a small number of candidates - and not necessarily cost, or housecleaning for that matter, as stated in the editorial.

Each Specialty Committee was invited to respond in writing to questions relating to their connection to other disciplines, core knowledge and organizational models. In addition, each of the
Specialty Committees was invited to attend a meeting of the Committee of Specialties to provide the Committee with a formal presentation and respond to additional questions. After considering each response to the above questions, applying the criteria, analysis and extensive consultation with stakeholders including the appropriate National Specialty Societies, Regional Advisory Committees, program directors and Chairs, the Committee of Specialties makes a recommendation for consideration by the Education Committee and ultimately the RCPSC Council.

\section{NEUROPATHOLOGY IS NOT A PRIMARY SPECIALTY}

1. Applying the RCPSC definition of a specialty, Neuropathology does not meet the criteria of a primary specialty:

- While currently residents can enter Neuropathology in their PGY-1 year, only 22 have been matched into Neuropathology over the past twelve years. ${ }^{1}$

- Neuropathologists are based primarily in large tertiary care centers.

- The ability to produce a valid and reliable examination is questionable with only one or two candidates per year.

- Neuropathology is not a foundation for additional competencies (subspecialties) recognized by the RCPSC.

- Neuropathology is not a broad-based body of knowledge, (all tissues/organs) but rather a distinct body of knowledge focused exclusively on the nervous system.

2. Neuropathology is not recognized as a primary specialty in the United States, Australia or Great Britain.

- In fact, Neuropathology is not recognized as either a specialty or subspecialty in Australia, arguably Canada's closest demographic comparator.

- Neuropathology is recognized in Great Britain and the United States as a subspecialty.

\section{NEUROPATHOLOGY IS A SUBSPECIALTY}

3. Neuropathology is a distinct body of knowledge that builds upon the broad-based body of knowledge defined in the specialty of Anatomical Pathology.

A comparison of the Objectives of Training and Specialty Training Requirements for both Anatomical Pathology and Neuropathology clearly demonstrates overlap and duplication between the disciplines. Almost $90 \%$ of the specific knowledge required under the "Medical Expert" role for Anatomical Pathology is identical to the "Medical Expert" role for Neuropathology. The basic clinical training year has not been deleted, rather it remains as a requirement of both disciplines. In addition, both Anatomical Pathology and Neuropathology 
require one year of approved residency selected from the same variety of options (e.g. one year of clinical or basic research, or six months of training in basic science). Of the remaining three years of training, specific training in Neuropathology (distinct from training requirements within Anatomical Pathology) totals 15-18 months.

Given the duplication between the Objectives of Training and Specialty Training Requirements for Anatomical Pathology and Neuropathology, it is clear that Neuropathology is a distinct body of knowledge, which builds on the foundation of Anatomical Pathology and appears to require approximately 18 months of discrete training in Neuropathology as currently indicated by the Specialty Committee's own document. (The Objectives of Training and Specialty Training Requirements can be viewed on the RCPSC web site at http://rcpsc.medical.org)

\section{Career paths must remain open}

It is precisely for this reason that multiple entry points into the subspecialty of Neuropathology are being considered. While it is not true that the majority of Neuropathologists have had entry via Neurology or Neurosurgery, (there has been only one in the past 12 years $)^{1}$ it is absolutely true that many interfaces exist with Neuropathology. Neuropathology as a subspecialty would facilitate flexibility of career choice in a way that is not possible through the current system of entry directly from medical school into a PGY-1 year in Neuropathology.

\section{Excellence in Neuropathology}

National Neuropathology meetings can continue to thrive, regardless of whether or not the discipline is recognized by the RCPSC as a specialty or subspecialty. Canada's reputation for excellence in Neuropathology would not change. In fact, the intent of the RCPSC is that the field of Neuropathology will be enhanced. With the development of a two to three year core base of training that capitalizes on the overlap which already exists between Anatomical Pathology and Neuropathology and increased flexibility of multiple entry routes into a subspecialty, there is the potential to attract more than the current one or two residents a year to the discipline.

Finally, there is considerable concern within the medical education community that the number and type of specialties with entry from the PGY-1 year sway medical students into early career decisions, early streaming into electives and increased competitiveness within the CaRMS match. $2,{ }^{3}$ Given Neuropathology's small numbers of residents in training, overlap with other disciplines within the Neurosciences, and specific knowledge built on a foundation of Anatomical Pathology Neuropathology as a subspecialty makes a great deal of sense.

Nadine Valk Education Policy Analyst

Robert H. A. Haslam Vice-President Education Royal College of Physicians and Surgeons of Canada.

\section{REFERENCES}

1. Data from the Canadian Post-M.D. Education Registry (CAPER)

2. Rich P. Restore flexibility in postgraduate education, MDs beg. Can Med Assoc J 2003; 169: 594-a.

3. Rich P. Students worried PGY-1 changes could mean more training time. Can Med Assoc J 2003; 169: 948. 